\title{
Transversality theorem in o-minimal structures
}

\author{
Ta Lê Loi
}

\begin{abstract}
In this paper we present Thom's transversality theorem in o-minimal structures (a generalization of semialgebraic and subanalytic geometry). There are no restrictions on the differentiability class and the dimensions of manifolds involved in comparison with the general case.
\end{abstract}

\section{Introduction}

In this paper we present Thom's transversality theorem for maps and sets that are definable in o-minimal structures (see the definition below). Whitney's paper [Whi35] provides examples of functions which are nonconstant on a connected set of critical points (see also examples in [Yom91]). Therefore, owing to Morse-Sard's theorem, in the general case (see [Lev71]) and also in the $\mathcal{X}$-version of the theorem given by Shiota [Shi97], there are some restrictions on the differentiability class and the dimensions of manifolds involved. In o-minimal structures, however, the theorem holds for any $C^{1}$-definable submanifolds of any dimensions of the jet spaces. This can be seen as an example of the ominimality being tame. Our proof is quite elementary, using some standard arguments of singularity theory in the o-minimal setting, and a tricky computation of the rank of the Jacobian matrix (see Lemma 2).

\section{O-minimal structures}

We only recall here the axioms of o-minimal structures. It is a generalization of semialgebraic and subanalytic geometry. We refer the reader to [VdD97], [VdDM96], [Cos00] and [Wil99] for many interesting o-minimal structures, and for many finiteness properties and tameness features of the o-minimality.

A structure on the real field $(\mathbb{R},+, \cdot)$ is a sequence $\mathcal{D}=\left(\mathcal{D}_{n}\right)_{n \in \mathbb{N}}$ such that the following conditions are satisfied for all $n \in \mathbb{N}$ :

- $\mathcal{D}_{n}$ is a Boolean algebra of subsets of $\mathbb{R}^{n}$;

- if $A \in \mathcal{D}_{n}$, then $A \times \mathbb{R}$ and $\mathbb{R} \times A \in \mathcal{D}_{n+1}$;

- if $A \in \mathcal{D}_{n+1}$, then $\pi(A) \in \mathcal{D}_{n}$, where $\pi: \mathbb{R}^{n+1} \rightarrow \mathbb{R}^{n}$ is the projection on the first $n$ coordinates;

- $\mathcal{D}_{n}$ contains $\left\{x \in \mathbb{R}^{n}: P(x)=0\right\}$, for every polynomial $P \in \mathbb{R}\left[X_{1}, \ldots, X_{n}\right]$.

A structure $\mathcal{D}$ is said to be $o$-minimal if:

- each set in $\mathcal{D}_{1}$ is a finite union of intervals and points.

A set belonging to $\mathcal{D}$ is said to be definable (in that structure). Definable maps in a structure $\mathcal{D}$ are maps whose graphs are definable sets in $\mathcal{D}$.

Received 10 August 2007, accepted in final form 9 January 2008, published online 22 July 2008.

2000 Mathematics Subject Classification 32B20, 58C27, 14P10.

Keywords: o-minimal structures, transversality.

This journal is (C) Foundation Compositio Mathematica 2008. 
In this note we fix an o-minimal structure on $(\mathbb{R},+, \cdot)$. By 'definable' we mean definable in this structure. Let $p$ be a positive integer, $p \geqslant 1$.

\section{Definable Whitney topology}

Let $N, M$ be $C^{p}$-definable submanifolds of $\mathbb{R}^{n}, \mathbb{R}^{m}$, respectively. Let $\mathcal{D}^{p}(N, M)$ denote the set of $C^{p}$-definable mappings from $N$ to $M$. On this space the definable Whitney topology is defined as follows (see [Esc02] or [Shi87]).

First consider the case $M=\mathbb{R}$. Let $v_{1}, \ldots, v_{s}$ be $C^{p-1}$-definable vector fields on $N$, such that $v_{1}(x), \ldots, v_{s}(x)$ span the tangent space $T_{x} N$ of $N$ at each $x \in N$. For each $f \in \mathcal{D}^{p}(N, \mathbb{R})$, and positive continuous definable function $\varepsilon$ on $N$, the $\varepsilon$-neighborhood of $f$ in this topology is defined by

$$
U_{\varepsilon}(f)=\left\{g \in \mathcal{D}^{p}(N, \mathbb{R}):\left|v_{i_{1}} \cdots v_{i_{k}}(g-f)\right|<\varepsilon, 1 \leqslant k \leqslant p, 1 \leqslant i_{1}, \ldots, i_{k} \leqslant s\right\},
$$

where $v f$ is the derivative of $f$ along $v$, that is, $v f(x)=d f(x)(v(x))$. Note that this topology does not depend on the choice of $v_{1}, \ldots, v_{s}$.

The topology on $\mathcal{D}^{p}\left(N, \mathbb{R}^{m}\right)=\mathcal{D}^{p}(N, \mathbb{R}) \times \cdots \times \mathcal{D}^{p}(N, \mathbb{R})$ is the product topology. For the general case, $\mathcal{D}^{p}(N, M)$ is a subspace of $\mathcal{D}^{p}\left(N, \mathbb{R}^{m}\right)$ with induced topology.

In this topology we have the following propositions which are proved in [Esc02].

Proposition 1. Let $T_{N}$ be a definable open neighborhood of $N$ in $\mathbb{R}^{n}$. Then the restriction map

$$
\mathcal{D}^{p}\left(T_{N}, M\right) \rightarrow \mathcal{D}^{p}(N, M),\left.\quad f \mapsto f\right|_{N}
$$

is continuous.

Proposition 2. Let $T_{M}$ be a $C^{p}$-definable submanifold of $\mathbb{R}^{m}$. Let $\pi: M \rightarrow T_{M}$ be a $C^{p}$-definable mapping. Then the mapping

$$
\mathcal{D}^{p}\left(N, T_{M}\right) \rightarrow \mathcal{D}^{p}(N, M), \quad f \mapsto \pi \circ f
$$

is continuous.

\section{Definable jet bundles}

Let $N, M$ be $C^{p}$-definable submanifolds of $\mathbb{R}^{n}, \mathbb{R}^{m}$, respectively. Let $0<r \leqslant p$. Let $J^{r}(N, M)$ denote the space of all $r$-jets of maps from $N$ to $M$ (see, for example, [Hir76] for the definition). We define the definable $r$-jet space by

$$
J_{\mathcal{D}}^{r}(N, M)=\left\{j^{r} f \in J^{r}(N, M): f \in \mathcal{D}^{r}(N, M)\right\} .
$$

To see that this space is a definable set, we can construct it as follows (cf. [Shi97]). Let $P^{r}\left(\mathbb{R}^{n}\right.$ ) denote the set of all polynomials in $n$ variable of degree at most $r$ which have their constant term equal to zero. Let

$$
R=\#\left\{\alpha=\left(\alpha_{1}, \ldots, \alpha_{n}\right) \in \mathbb{N}^{n}: 1 \leqslant|\alpha|=\alpha_{1}+\cdots+\alpha_{n} \leqslant r\right\} .
$$

We identify $P^{r}\left(\mathbb{R}^{n}\right)$ with $\mathbb{R}^{R}$, by

$$
\sum_{1 \leqslant|\alpha| \leqslant r} a_{\alpha} X^{\alpha} \leftrightarrow\left(\alpha ! a_{\alpha}\right)_{1 \leqslant|\alpha| \leqslant r}
$$

For the case $N=U$ and $M=V$ being open subsets of $\mathbb{R}^{n}$ and $\mathbb{R}^{m}$, respectively, it is easy to see that

$$
J_{\mathcal{D}}^{r}(U, V)=J^{r}(U, V) \equiv U \times V \times \prod_{i=1}^{m} P^{r}\left(\mathbb{R}^{n}\right) \equiv U \times V \times \mathbb{R}^{m R} .
$$




\section{TRANSVERSALITY THEOREM IN O-MINIMAL STRUCTURES}

For $f \in \mathcal{D}^{r}(U, V)$, we denote and identify the $r$-jet of $f$ at $x$ by

$$
j^{r} f(x)=\left(x, f(x), \quad \sum_{1 \leqslant|\alpha| \leqslant r} \frac{\partial^{\alpha} f(x)}{\alpha !}(X-x)^{\alpha}\right) \equiv\left(x, f(x), \partial^{\alpha} f(x)\right)_{1 \leqslant|\alpha| \leqslant r},
$$

where

$$
\partial^{\alpha} f=\frac{\partial^{|\alpha|} f}{\partial x_{1}^{\alpha_{1}} \cdots \partial x_{n}^{\alpha_{n}}}
$$

when $\alpha=\left(\alpha_{1}, \ldots, \alpha_{n}\right) \in \mathbb{N}^{n}$.

For general $N$ and $M$, take $C^{r}$-definable tubular neighborhoods $\left(T_{N}, \pi_{N}, \rho_{N}\right)$ and $\left(T_{M}, \pi_{M}, \rho_{M}\right)$ of $N$ and $M$ in $\mathbb{R}^{n}$ and $\mathbb{R}^{m}$, respectively. Note that such neighborhoods exist by [Esc02, Theorem 1.9]. Then we have

$$
\begin{aligned}
J_{\mathcal{D}}^{r}(N, M) & =\left\{j^{r}\left(\pi_{M} \circ f \circ \pi_{N}\right)(x): f \in \mathcal{D}^{r}\left(T_{N}, T_{M}\right), x \in N\right\} \\
& =\left\{j^{r}\left(\pi_{M} \circ T_{x}^{r} f \circ \pi_{N}\right)(x): f \in J^{r}\left(T_{N}, T_{M}\right), x \in N\right\},
\end{aligned}
$$

where

$$
T_{x}^{r} f(y)=f(x)+\sum_{1 \leqslant|\alpha| \leqslant r} \frac{\partial^{\alpha} f(x)}{\alpha !}(y-x)^{\alpha} .
$$

Indeed, for each $f \in \mathcal{D}^{r}(N, M)$, there exists $\tilde{f}=f \circ \pi_{N} \in \mathcal{D}^{r}\left(T_{N}, T_{M}\right)$ such that $\left.\tilde{f}\right|_{N}=f$, so we have the first equality. By Leibniz's rule, $\partial^{\alpha}\left(\pi_{M} \circ f \circ \pi_{N}\right)(x)$ is a polynomial of $\partial^{\beta} \pi_{N}(x), \partial^{\delta} f\left(\pi_{N}(x)\right)$ and $\partial^{\gamma} \pi_{M}\left(f\left(\pi_{N}(x)\right)\right)$, with $|\beta|,|\delta|,|\gamma| \leqslant|\alpha|$, so we have the second equality.

Hence, $J_{\mathcal{D}}^{r}(N, M)$ is a definable submanifold of $J^{r}\left(\mathbb{R}^{n}, \mathbb{R}^{m}\right)$.

\section{Definable stratifications}

A definable $C^{p}$ stratification of $X \subset \mathbb{R}^{m}$ is a partition $\mathcal{S}$ of $X$ into finitely many subsets, called strata, such that:

- each stratum is a $C^{p}$ submanifold of $\mathbb{R}^{m}$ and also a definable set;

- for every $S \in \mathcal{S}, \bar{S} \backslash S$ is a union of some of the strata.

We say that $\mathcal{S}$ satisfies Whitney's condition $(a)$ if the following holds. For every $S, R \in \mathcal{S}$ with $S \subset \bar{R}$, given a sequence of points $\left(x_{k}\right)$ in $R$ converging to a point $y$ of $S$ such that $T_{x_{k}} R$ converges to a vector subspace $T$ of $\mathbb{R}^{m}$, we have $T_{y} S \subset T$.

\section{Morse-Sard's theorem}

Theorem 1 (Morse-Sard). Let $N$ and $M$ be $C^{p}$ definable manifolds, and $f: N \rightarrow M$ be a $C^{p}$ definable map. For each $s \in \mathbb{N}$, let

$$
\Sigma_{s}(f)=\{x \in N: \operatorname{rank} d f(x)<s\} \quad \text { and } \quad C_{s}(f)=f\left(\Sigma_{s}(f)\right) .
$$

Then $C_{s}(f)$ is definable and $\operatorname{dim} C_{s}(f)<s$.

Proof (Cf. [Wil99, Theorem 2.7]). It is easy to see that $C_{s}(f)$ is definable. To prove the second part we suppose, contrary to the assertion, that $\operatorname{dim} C_{s}(f) \geqslant s$. Then, by the definable choice [VdDM96, Theorem 4.5], there exists a definable subset $U$ of $C_{s}(f)$ and a definable $C^{p}$ mapping $s: U \rightarrow \Sigma_{s}(f)$ such that $f \circ s=i d_{U}$. So rank $d f(s(y)) d s(y) \geqslant s$, for all $y \in U$. Hence, rank $d f(x) \geqslant s$, for all $x \in s(U)$. This is a contradiction.

Note that, in general, Morse-Sard's theorem [Sard42] requires the differentiability class of $f$, namely $p>\max (\operatorname{dim} N-\operatorname{dim} M, 0)$ if $s=\operatorname{dim} M$. See [Whi35] and [Yom91] for examples where the 
theorem does not hold for mappings of low smoothness. However, by Theorem 1, Thom's transversality theorem in o-minimal context holds for any $C^{1}$-definable submanifolds of any dimensions of the jet spaces. For the restrictions in the general case we refer the readers to [Lev71, 7. Theorem 1] and [Shi97, Theorem II.5.4(3)].

\section{Transversality theorem}

Theorem 2 (Transversality). Let $N$ and $M$ be definable $C^{p}$ manifolds. Let $\mathcal{A}$ be a finite collection of definable $C^{1}$ submanifolds of $J_{\mathcal{D}}^{r}(N, M)(0<r<p)$. Then the set

$$
\tau_{r}(\mathcal{A})=\left\{f \in \mathcal{D}^{p}(N, M): j^{r} f \text { is transverse to each member of } \mathcal{A}\right\}
$$

is a dense subset of $\mathcal{D}^{p}(N, M)$.

Moreover, if $\mathcal{A}$ is a stratification of a closed subset and satisfies Whitney's condition (a), then $\tau_{r}(\mathcal{A})$ is an open subset of $\mathcal{D}^{p}(N, M)$.

To prove the theorem, we use the following lemmas.

Lemma 1. Let $U$ be an open definable subset of $\mathbb{R}^{n}$. Let $\varepsilon: U \rightarrow \mathbb{R}$ be a positive continuous definable function. Then there exists a positive definable $C^{p}$ function $\varphi: U \rightarrow \mathbb{R}$, such that

$$
\left|\partial^{\alpha} \varphi\right|<\varepsilon, \quad \text { for all }|\alpha| \leqslant p .
$$

Proof. The proof is a modified version of the proof of [Loi06, Lemma 4].

For $n=1$. By finiteness of the number of the connected components of $U$, we can assume $U=(a, b)$. Moreover, by cell decomposition [VdDM96, Theorem 4.2], $\varepsilon$ can be smoothed at unsmooth points in an elementary way. So we have reduced this to the case that $\varepsilon$ is of class $C^{p}$.

If $\lim _{t \rightarrow a^{+}} \varepsilon(t)>0$ and $\lim _{t \rightarrow b^{-}} \varepsilon(t)>0$, then take $\varphi=c$, where $c$ is a constant, $0<c<$ $\min _{t \in(a, b)} \varepsilon(t)$.

If $\lim _{t \rightarrow b^{-}} \varepsilon(t)=0$, then by motonicity [VdDM96, Theorem 4.1], $\varepsilon^{\prime}$ is strictly increasing on a neighborhood of $b$, and tends to zero at $b$. Repeating the previous argument for $\varepsilon^{\prime \prime}, \ldots, \varepsilon^{(p)}$, we obtain $a<b^{\prime}<b$, such that $\varepsilon, \varepsilon^{\prime}, \ldots, \varepsilon^{(p)}$ are strictly monotone on $\left(b^{\prime}, b\right)$, tend to zero at $b$, and $|\varepsilon(t)|<1, \ldots,\left|\varepsilon^{(p)}(t)\right|<1$, for all $t>b^{\prime}$. Similarly for the case $\lim _{t \rightarrow a^{+}} \varepsilon(t)=0$, the above conditions are satisfied for $\varepsilon$ on $\left(a, a^{\prime}\right)$, with $a<a^{\prime}<b$. If we take a constant $M$ that is large enough, then $\varphi=\varepsilon^{p+1} / M$ has the desired properties.

For general $n$. Let

$$
\varepsilon_{1}(x)=\frac{\varepsilon(x)}{N\left(1+\|x\|^{p}\right)}
$$

where $N$ is a positive number. Fix $x_{0} \in U$. For $t \in \mathbb{R}$, let

$$
\alpha(t)=\min \left\{\varepsilon_{1}(x):\left\|x-x_{0}\right\|^{2} \leqslant t^{2}, d(x, \partial U) \geqslant \frac{d\left(x_{0}, \partial U\right)}{1+t^{2}}\right\} .
$$

Applying the case $n=1$, we have a positive definable $C^{p}$ function $g: \mathbb{R} \rightarrow \mathbb{R}$, such that $\left|g^{(k)}\right|<\alpha$, for all $k \in\{0, \ldots, p\}$. Now let $\varphi(x)=g\left(\|x\|^{2}\right)$. Then by the chain rule, when $N$ is large enough, we have $\left|\partial^{\alpha} \varphi(x)\right|<N \varepsilon_{1}(x)\|x\|^{|\alpha|}<\varepsilon(x)$, for all $x \in U$, for all $|\alpha|<p$.

Lemma 2. Let $\varphi: \mathbb{R}^{n} \rightarrow \mathbb{R}$ be a $C^{p}$ function, and $\alpha \in \mathbb{N}^{n}$. For $\gamma \in \mathbb{N}^{n}$, denote $\gamma \leqslant \alpha$ if and only if $\alpha-\gamma \in \mathbb{N}^{n}$, and $\gamma<\alpha$ if and only if $\gamma \leqslant \alpha, \gamma \neq \alpha$. Then there exist real numbers $a_{\gamma}(\gamma<\alpha)$ such 


\section{TRANSVERSALITY THEOREM IN O-MINIMAL STRUCTURES}

that for all $\beta \in \mathbb{N}^{n}$ with $|\beta| \leqslant|\alpha|$, we have

$$
\begin{aligned}
& \partial^{\beta}\left(x^{\alpha} \varphi\right)=\sum_{\gamma<\alpha} a_{\gamma} x^{\alpha-\gamma} \partial^{\beta}\left(x^{\gamma} \varphi\right) \quad \text { if } \beta \neq \alpha, \\
& \partial^{\alpha}\left(x^{\alpha} \varphi\right)=\alpha ! \varphi+\sum_{\gamma<\alpha} a_{\gamma} x^{\alpha-\gamma} \partial^{\alpha}\left(x^{\gamma} \varphi\right) .
\end{aligned}
$$

Proof. First, note that

$$
\partial^{\delta}\left(x^{\alpha}\right)= \begin{cases}\frac{\alpha !}{(\alpha-\delta) !} x^{\alpha-\delta} & \text { if } \delta \leqslant \alpha, \\ 0 & \text { if } \delta \nless \alpha .\end{cases}
$$

Let $a_{\gamma}(\gamma<\alpha)$ be the solution of the following system of linear equations of triangular form:

$$
\sum_{\delta \leqslant \gamma<\alpha} \frac{(\gamma-\delta) !}{\gamma !} a_{\gamma}=\frac{\alpha !}{(\alpha-\delta) !} \quad\left(\delta \in \mathbb{N}^{n}, \delta<\alpha\right) .
$$

We shall check that the $a_{\gamma}(\gamma<\alpha)$ satisfy the equations of the lemma.

For each $\beta \in \mathbb{N}^{n},|\beta| \leqslant|\alpha|$, by Leibniz's rule and the note, the left-hand side is

$$
A_{\beta}=\partial^{\beta}\left(x^{\alpha} \varphi\right)=\sum_{\delta \leqslant \beta} C_{\beta}^{\delta} \partial^{\beta}\left(x^{\alpha}\right) \partial^{\beta-\delta} \varphi=\sum_{\delta \leqslant \beta, \delta \leqslant \alpha}\left(C_{\beta}^{\delta} \frac{\alpha !}{(\alpha-\delta) !} x^{\alpha-\delta}\right) \partial^{\beta-\delta} \varphi .
$$

On the other hand, by Leibniz's rule and the note, the right-hand side is

$$
\begin{aligned}
B_{\beta} & =\sum_{\gamma<\alpha} a_{\gamma} x^{\alpha-\gamma} \partial^{\beta}\left(x^{\gamma} \varphi\right) \\
& =\sum_{\gamma<\alpha} a_{\gamma} x^{\alpha-\gamma}\left(\sum_{\delta \leqslant \beta} C_{\beta}^{\delta} \partial^{\delta}\left(x^{\gamma}\right) \partial^{\beta-\delta} \varphi\right) \\
& =\sum_{\delta \leqslant \beta}\left(\sum_{\gamma<\alpha} a_{\gamma} x^{\alpha-\gamma} C_{\beta}^{\delta} \partial^{\delta}\left(x^{\gamma}\right)\right) \partial^{\beta-\delta} \varphi \\
& =\sum_{\delta \leqslant \beta}\left(\sum_{\delta \leqslant \gamma<\alpha} a_{\gamma} x^{\alpha-\gamma} C_{\beta}^{\delta} \partial^{\delta}\left(x^{\gamma}\right)\right) \partial^{\beta-\delta} \varphi \\
& =\sum_{\delta \leqslant \beta}\left(\sum_{\delta \leqslant \gamma<\alpha} C_{\beta}^{\delta} \frac{(\gamma-\delta) !}{\gamma !} a_{\gamma} x^{\alpha-\delta}\right) \partial^{\beta-\delta} \varphi .
\end{aligned}
$$

By the definition of the $a_{\gamma}$, we have

$$
B_{\beta}=\sum_{\delta \leqslant \beta, \delta<\alpha}\left(C_{\beta}^{\delta} \frac{\alpha !}{(\alpha-\delta) !} x^{\alpha-\delta}\right) \partial^{\beta-\delta} \varphi .
$$

If $|\beta| \leqslant|\alpha|$ and $\beta \neq \alpha$, then

$$
\left\{\delta \in \mathbb{N}^{n}: \delta \leqslant \beta, \delta \leqslant \alpha\right\}=\left\{\delta \in \mathbb{N}^{n}: \delta \leqslant \beta, \delta<\alpha\right\},
$$

and, thus, $A_{\beta}=B_{\beta}$.

If $\beta=\alpha$, then

$$
\left\{\delta \in \mathbb{N}^{n}: \delta \leqslant \beta, \delta \leqslant \alpha\right\} \backslash\left\{\delta \in \mathbb{N}^{n}: \delta \leqslant \beta, \delta<\alpha\right\}=\{\alpha\},
$$

and thus

$$
A_{\alpha}-B_{\alpha}=C_{\alpha}^{\alpha} \frac{\alpha !}{(\alpha-\alpha) !} x^{\alpha-\alpha} \partial^{\alpha-\alpha} \varphi=\alpha ! \varphi
$$

This completes the proof of the lemma. 
Lemma 3. Let $N, J$ and $T$ be definable $C^{p}$ manifolds, and $\Phi: T \times N \rightarrow J$ be a definable $C^{p}$ map. Let $\mathcal{A}$ be a finite collection of definable submanifolds of $J$. If $\Phi$ is submersive, then the set

$$
\tau(\Phi, \mathcal{A})=\{t \in T: \Phi(t, \cdot) \text { is transverse to each member of } \mathcal{A}\}
$$

is a definable set and $\operatorname{dim}(T \backslash \tau(\Phi, \mathcal{A}))<\operatorname{dim} T$.

Proof. Use Morse-Sard's theorem (see [Loi06, Proposition 2] for details).

Proof of Theorem 2 (Cf. [Shi97, Theorem II.5.4(3)]). We reduce the proof to the case $N=U$ being an open subset of $\mathbb{R}^{n}$, and $M=\mathbb{R}^{m}$, by the following arguments.

Let $\left(T_{M}, \pi_{M}, \rho_{M}\right)$ be a $C^{p}$-definable tubular neighborhood of $M$ in $\mathbb{R}^{m}$. Let $\pi_{M *}: J_{\mathcal{D}}^{r}\left(N, T_{M}\right) \rightarrow$ $J_{\mathcal{D}}^{r}(N, M), j^{r} f \mapsto j^{r}\left(\pi_{M} \circ f\right)$. Let $f \in \mathcal{D}^{p}(N, M)$. If $g$ is an approximation of $f$ in $\mathcal{D}^{p}\left(N, \mathbb{R}^{m}\right)$ and $j^{r} g$ is transverse to $\pi_{M *}^{-1}(A)$, for each $A \in \mathcal{A}$, then, by Proposition $2, \pi_{M} \circ g$ is an approximation of $f$ in $\mathcal{D}^{p}(N, M)$ and $j^{r}\left(\pi_{M} \circ g\right)$ is transverse to each member of $\mathcal{A}$. Therefore, we can reduce the proof to the case $M=\mathbb{R}^{m}$.

On the other hand, let $\left(T_{N}, \pi_{N}, \rho_{N}\right)$ be a $C^{p}$-definable tubular neighborhood of $N$ in $\mathbb{R}^{n}$. For each $A \in \mathcal{A}$ being a definable submanifold of $J_{\mathcal{D}}^{r}\left(N, \mathbb{R}^{m}\right)$, define

$$
\tilde{A}=\left\{\left(x, y, a_{\alpha}\right)_{1 \leqslant|\alpha| \leqslant r} \in J^{r}\left(T_{N}, \mathbb{R}^{m}\right):\left(\pi_{N}(x), y, a_{\alpha}\right)_{1 \leqslant|\alpha| \leqslant r} \in A\right\} .
$$

Then $\tilde{A}$ is a definable submanifold of $J^{r}\left(T_{N}, \mathbb{R}^{m}\right)$. Let $f \in \mathcal{D}^{p}\left(N, \mathbb{R}^{m}\right)$. If $g$ is an approximation of $f$ in $\mathcal{D}^{p}\left(T_{N}, \mathbb{R}^{m}\right)$ and $j^{r} g$ is transverse to $\tilde{A}$, for each $A \in \mathcal{A}$, then, by Proposition $1,\left.g\right|_{N}$ is an approximation of $f$ in $\mathcal{D}^{p}\left(N, \mathbb{R}^{m}\right)$ and $j^{r}\left(\left.g\right|_{N}\right)$ is transverse to each member of $\mathcal{A}$ in $J_{\mathcal{D}}^{r}\left(N, \mathbb{R}^{m}\right)$. Therefore, we can reduce the proof to the case where $N$ is an open subset of $\mathbb{R}^{n}$.

Now, let $f: U \rightarrow \mathbb{R}^{m}$ and let $f=\left(f_{1}, \ldots, f_{m}\right)$ be a $C^{p}$-definable map. Let $\varepsilon: U \rightarrow \mathbb{R}$ be a positive continuous definable function. We construct a function in $\tau_{r}(\mathcal{A})$ which is in a $\varepsilon$-neigborhood of $f$.

Case $m=1$. Let $P=\#\left\{\alpha \in \mathbb{N}^{n}:|\alpha| \leqslant p\right\}$ and $C=P^{2}(p !)^{2 n}$. Let $\varphi: U \rightarrow \mathbb{R}$ be a positive $C^{p}$-definable function such that

$$
\left|\partial^{\alpha} \varphi(x)\right|<\frac{\varepsilon(x)}{C\left(1+\|x\|^{p}\right)}, \quad \text { for all }|\alpha| \leqslant p .
$$

Note that such a function exists by Lemma 1.

Consider the following family of definable functions

$$
F(t, x)=f_{t}(x)=f(x)+\sum_{|\alpha| \leqslant r} t_{\alpha} x^{\alpha} \varphi(x),
$$

where $I=(-1,1), t=\left(t_{\alpha}\right)_{|\alpha| \leqslant r} \in I^{R_{0}}, R_{0}=\#\left\{\alpha \in \mathbb{N}^{n}:|\alpha| \leqslant r\right\}$ and $x \in U$. First, we prove that $f_{t}$ is in a $\varepsilon$-neighborhood of $f$. Indeed, for each $\alpha \in \mathbb{N}^{n}$ with $|\alpha| \leqslant p$,

$$
\left|\partial^{\alpha}\left(f_{t}-f\right)(x)\right| \leqslant \sum_{|\beta| \leqslant r}\left|t_{\beta}\right|\left|\partial^{\alpha}\left(x^{\beta} \varphi\right)(x)\right| \leqslant \sum_{|\beta| \leqslant r}\left|\partial^{\alpha}\left(x^{\beta} \varphi\right)(x)\right| .
$$

From the proof of Lemma 2 and by the definition of $\varphi$, we have

$$
\begin{aligned}
\left|\partial^{\alpha}\left(x^{\beta} \varphi\right)\right|=\left|\sum_{\delta \leqslant \alpha} C_{\alpha}^{\delta} \partial^{\delta}\left(x^{\beta}\right) \partial^{\alpha-\delta} \varphi\right| & =\left|\sum_{\delta \leqslant \beta, \delta \leqslant \alpha} C_{\alpha}^{\delta} \frac{\beta !}{(\beta-\delta) !} x^{\beta-\delta} \partial^{\alpha-\delta} \varphi\right| \\
& <\sum_{|\delta| \leqslant p}(p !)^{n}(p !)^{n}\left(1+\|x\|^{p}\right) \frac{\varepsilon}{C\left(1+\|x\|^{p}\right)} .
\end{aligned}
$$

Therefore, by the selection of $C$, we obtain

$$
\left|\partial^{\alpha}\left(f_{t}-f\right)(x)\right|<\varepsilon(x), \quad \text { for all } x \in U .
$$




\section{TRANSVERSALITY THEOREM IN O-MINIMAL STRUCTURES}

Using Lemma 3, we prove that there are many $t \in I^{R_{0}}$, such that $f_{t} \in \tau_{r}(\mathcal{A})$. To this end, we need to check that

$$
\Phi: I^{R_{0}} \times U \rightarrow J^{r}(U, \mathbb{R}) \equiv U \times \mathbb{R}^{R_{0}},
$$

defined by $\Phi(t, x)=\left(x,\left(\partial^{\alpha} f_{t}(x)\right)_{|\alpha| \leqslant r}\right)$, is a submersion.

Calculating, we can write the Jacobian matrix $J \Phi(x, t)$ in block form:

$$
\left(\begin{array}{ccccccccc}
O & \cdots & O & \cdots & O & \cdots & O & \cdots & I_{n} \\
\varphi(x) & \cdots & x^{\gamma} \varphi(x) & \cdots & x^{\beta} \varphi(x) & \cdots & x^{\alpha} \varphi(x) & \cdots & * \\
\vdots & & \vdots & & \vdots & & \vdots & & \vdots \\
\partial^{\gamma} \varphi(x) & \cdots & \partial^{\gamma} x^{\gamma} \varphi(x) & \cdots & \partial^{\gamma} x^{\beta} \varphi(x) & \cdots & \partial^{\gamma} x^{\alpha} \varphi(x) & \cdots & * \\
\vdots & & \vdots & & \vdots & & \vdots & & \vdots \\
\partial^{\beta} \varphi(x) & \cdots & \partial^{\beta} x^{\gamma} \varphi(x) & \cdots & \partial^{\beta} x^{\beta} \varphi(x) & \cdots & \partial^{\beta} x^{\alpha} \varphi(x) & \cdots & * \\
\vdots & & \vdots & & \vdots & & \vdots & & \vdots \\
\partial^{\alpha} \varphi(x) & \cdots & \partial^{\alpha} x^{\gamma} \varphi(x) & \cdots & \partial^{\alpha} x^{\beta} \varphi(x) & \cdots & \partial^{\alpha} x^{\alpha} \varphi(x) & \cdots & * \\
\vdots & & \vdots & & \vdots & & \vdots & & \vdots
\end{array}\right)
$$

where $1 \leqslant|\gamma|<|\beta|=|\alpha|$.

Applying Lemma 2, we can reduce the matrix to the form

$$
\left(\begin{array}{ccccccccc}
O & \cdots & O & \cdots & O & \cdots & O & \cdots & I_{n} \\
\varphi(x) & \cdots & 0 & \cdots & 0 & \cdots & 0 & \cdots & * \\
\vdots & & \vdots & & \vdots & & \vdots & & \vdots \\
\partial^{\gamma} \varphi(x) & \cdots & \gamma ! \varphi(x) & \cdots & 0 & \cdots & 0 & \cdots & * \\
\vdots & & \vdots & & \vdots & & \vdots & & \vdots \\
\partial^{\beta} \varphi(x) & \cdots & * & \cdots & \beta ! \varphi(x) & \cdots & 0 & \cdots & * \\
\vdots & & \vdots & & \vdots & & \vdots & & \vdots \\
\partial^{\alpha} \varphi(x) & \cdots & * & \cdots & 0 & \cdots & \alpha ! \varphi(x) & \cdots & * \\
\vdots & & \vdots & & \vdots & & \vdots & & \vdots
\end{array}\right) .
$$

Therefore, rank $J \Phi(t, x)=R_{0}+n$, for all $(t, x) \in I^{R_{0}} \times U$, that is, $\Phi$ is submersive.

For the general case. Use the same arguments as in the case $m=1$ for the family

$$
F(t, x)=\left(f_{1}(x)+\sum_{|\alpha| \leqslant r} t_{1, \alpha} x^{\alpha} \varphi(x), \ldots, f_{m}(x)+\sum_{|\alpha| \leqslant r} t_{m, \alpha} x^{\alpha} \varphi(x)\right),
$$

where $t=\left(t_{i, \alpha}\right)_{1 \leqslant i \leqslant m,|\alpha| \leqslant r} \in I^{m R_{0}}$.

Since the mapping $j^{r}: \mathcal{D}^{p}(N, M) \rightarrow \mathcal{D}^{p-r}\left(N, J_{\mathcal{D}}^{r}(N, M)\right)$ is continuous and

$$
\tau_{r}(\mathcal{A})=\left(j^{r}\right)^{-1}\left\{F \in \mathcal{D}^{p-r}\left(N, J_{\mathcal{D}}^{r}(N, M)\right): F \text { is transverse to each member of } \mathcal{A}\right\},
$$

the proof of the second part of the theorem reduces to the case where $\mathcal{A}$ is a stratification of a definable closed subset of $M$ and satisfying Whitney's condition $(a)$. Let $K$ be the subset of $J_{\mathcal{D}}^{1}(N, M)$ defined by $\alpha \in K$ if and only if there exist $x \in N, S \in \mathcal{A}, y \in S$ such that $\alpha_{x, y}$ is not transverse to $T_{y} S$, where $\alpha_{x, y}: T_{x} N \rightarrow T_{y} M$ denotes the restriction of $\alpha$ to $J_{\mathcal{D}}^{1}(N, M)_{(x, y)}$.

Then by the proof of [Fel65, Proposition 3.6], $K$ is closed in $J_{\mathcal{D}}^{1}(N, M)$. Since the mapping $j$ : $\mathcal{D}^{p}(N, M) \rightarrow J_{\mathcal{D}}^{1}(N, M), f \mapsto j^{1} f$, is continuous, $j^{-1}(K)$ is closed. Therefore, $\tau_{1}(\mathcal{A})=\mathcal{D}^{p}(N, M) \backslash$ $j^{-1}(K)$ is open. 


\section{TRANSVERSALITY THEOREM IN O-MINIMAL STRUCTURES}

Remark. One can check that the theorem holds in any real closed field. We do not know whether the theorem holds for $p=\infty$ or $p=\omega$.

\section{ACKNOWLEDGEMENTS}

Part of this paper was written at the Research Institute for Mathematical Sciences (RIMS), Kyoto University. The author thanks the institute and Professor Kyoji Saito for his invitation and support. Gratitude is also extended to Professor Masahiro Shiota for his kindness and many helpful suggestions during the preparation of the paper at the Graduate School of Mathematics, Nagoya University.

\section{REFERENCES}

Cos00 M. Coste, An introduction to o-minimal geometry, Dottorato di Ricerca in Matematica, Dip. Mat. Pisa, Instituti Editoriali e Poligrafici Internazionali (2000).

Esc02 J. Escribano, Approximation theorems in o-minimal structures, Illinois J. Math. 46 (2002), 111-128.

Fel65 E. A. Feldman, The geometry of immersion. I, Trans. Amer. Math. Soc. 120 (1965), 185-224.

Hir76 M. W. Hirsch, Differential topology (Springer, Berlin, 1976).

Lev71 H. J. Levine, Singularities of differentiable mappings, Lecture Notes in Mathematics, vol. 192 (Springer, Berlin, 1971), 1-89.

Loi06 T. L. Loi, Density of morse functions on sets definable in o-minimal structures, Ann. Polon. Math. 89 (2006), 289-299.

Sard42 A. Sard, The measure of the critical values of differentiable maps, Bull. Amer. Math. Soc. 48 (1942), 883-890.

Shi87 M. Shiota, Nash manifolds, Lecture Notes in Mathematics, vol. 1269 (Springer, Berlin, 1987).

Shi97 M. Shiota, Geometry of subanalytic and semialgebraic sets, Progress in Mathematics, vol. 150 (Birkhäuser, Boston, MA, 1997).

VdD97 L. van den Dries, Tame topology and o-minimal structures, London Mathematical Society Lecture Notes Series, vol. 248 (Cambridge University Press, Cambridge, 1997).

VdDM96 L. van den Dries and C. Miller, Geometric categories and o-minimal structures, Duke Math. J. 84 (1996), 497-540.

Whi35 H. Whitney, A function not constant on connected set of critical points, Duke Math. J. 1 (1935), $514-517$.

Wil99 A. J. Wilkie, A theorem of the complement and some new o-minimal structures, Selecta Math. (N.S.) 5 (1999), 397-421.

Yom91 Y. Yomdin, Surjective mappings whose differential is nowhere surjective, Proc. Amer. Math. Soc. 111 (1991), 267-270.

Ta Lê Loi taleloi@hotmail.com

Department of Mathematics, University of Dalat, Dalat, Vietnam 